\title{
Successful treatment by novel endoscopic ultrasound-guided devascularization for refractory splenic artery aneurysm associated with portal hypertension
}

Splenic artery aneurysm carries significant risk of rupture and life-threatening hemorrhage [1]. Therapy utilizing digital subtraction angiography (DSA) with embolization is usually safe and effective; however, incomplete occlusion can occur and frequently requires surgical repair [2]. We report the case of refractory splenic artery aneurysm associated with portal hypertension that was successfully treated by a novel endoscopic ultrasound (EUS)-guided devascularization technique.

A 64-year-old woman with chronic hepatitis B presented with epigastric abdominal pain for 4 months. Esophagogastro-

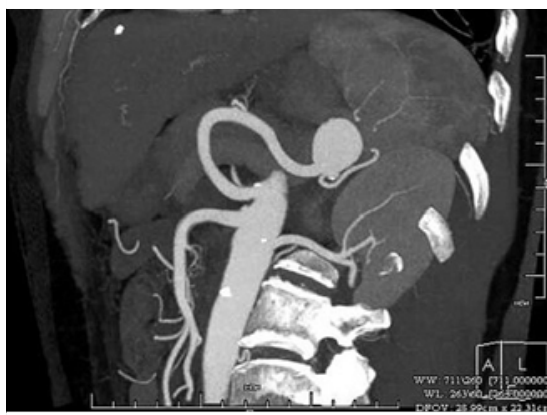

Fig. 1 Computed tomography revealed splenic artery aneurysm and cirrhosis with portal hypertension. duodenoscopy showed isolated gastric varix in the fundus. Computed tomography revealed splenic artery aneurysm and cirrhosis with portal hypertension ( $\triangleright$ Fig. 1). Embolization via DSA was performed successfully, as confirmed by selective splenic arteriography. One week later, a 2 -cm residual aneurysm cavity was detected by EUS (\$Fig.2). After EUS-guided obliteration of gastric varices, which hamper transgastric puncture to the aneurysm, a standard 22-gauge fine-needle aspiration needle was inserted into the aneurysmal neck, and a preloaded 0.018-inch fibered interlock detachable coil (F-IDC; 2D $12 \mathrm{~mm}, 30 \mathrm{~cm}$; Boston Scientific, Marlborough, Massachusetts, USA) was slowly pushed out of the needle tip into the residual aneurysmal cavity. After confirming that the aneurysmal neck was adequately coiled, the delivery wire was removed. EUS showed the direction of inflow had changed and the velocity had slowed down. Then, $3 \mathrm{~mL}$ lauromacrogol was injected into the aneurysmal sac; inflow slowed down further. Rapid injection of $0.5 \mathrm{~mL}$ tissue glue followed by $2 \mathrm{~mL}$ lauromacrogol generated a hyperechoic clot in the aneurysmal neck, with cessation of inflow ( Video 1). EUS with Color Doppler confirmed complete occlusion of the original aneurysm at the 8-week and 16-week follow-ups (> Fig.3).

Angiographic re-embolization of an aneurysm may not be feasible because of small vessel caliber [3]. The key to successful management of refractory aneurysm is selective devascularization of high-velocity blood inflow from the neck of the sac leading to the endoleak, rather than direct deployment of excess coils in the sac with risk of erosion and migration of coils $[4,5]$. With more powerful embolization effect and more control in selecting embolization sites, F-IDCs are the preferred treatment modality for vascular lesions with high blood flow that require highly precise embolization. Though not reported in the past, F-IDCs can be used successfully for EUS-guided aneurysm embolization. We therefore considered the EUS-guided selective devascularization with F-IDC, glue, and lauromacrogol to be a valuable therapeutic alternative for refractory cases with both splenic artery aneurysm and gastric varices.

Endoscopy_UCTN_Code_TTT_1AS_2AC
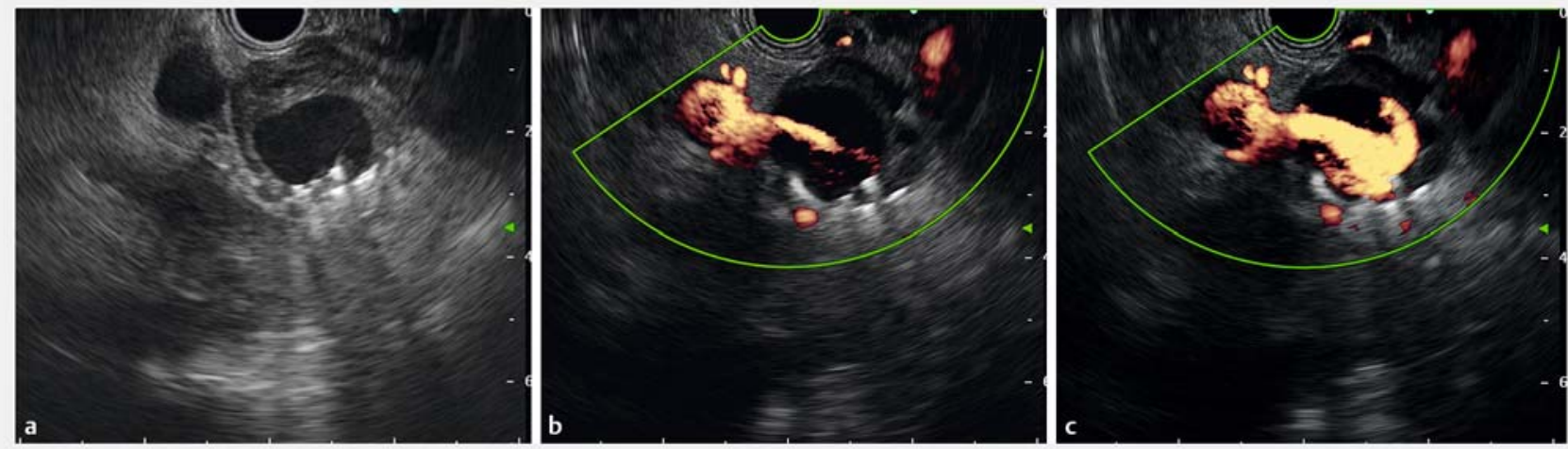

Fig. 2 Endoscopic ultrasound images. a A thick walled anechoic residual cavity with multiple hyperechoic coils was detected. b Color Doppler revealed the inflow from tortuous splenic artery. c Color Doppler showed the inflow and the retractable flow streams from the sac wall. 


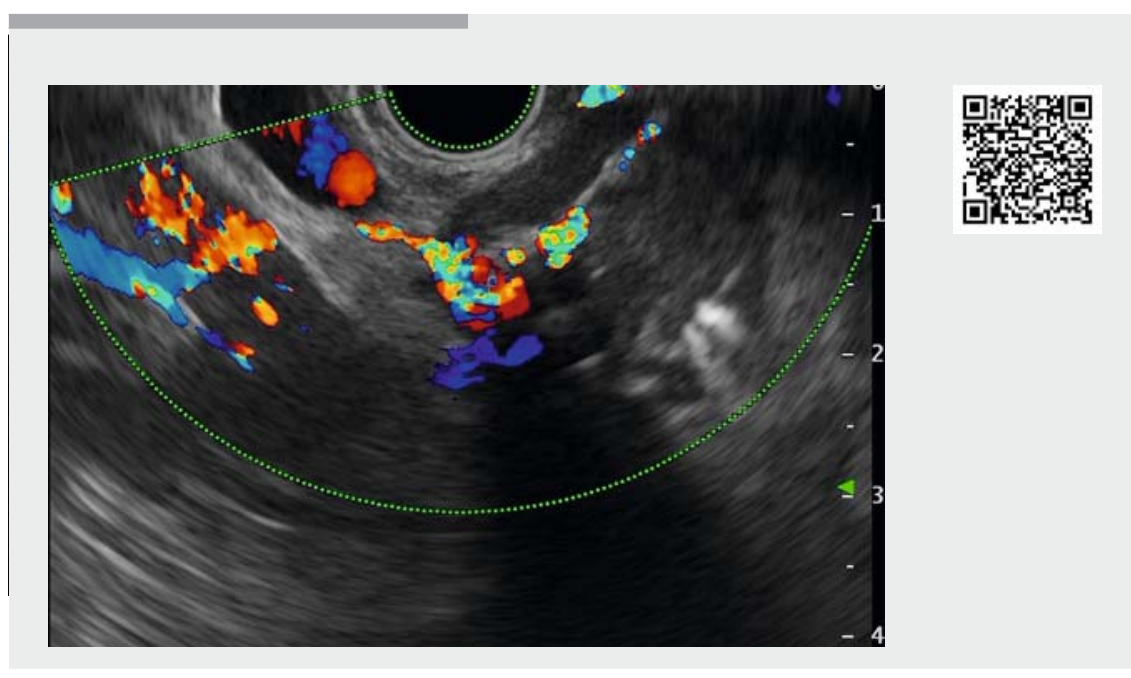

Video 1 Successful treatment by novel endoscopic ultrasound-guided devascularization for refractory splenic artery aneurysm associated with portal hypertension.

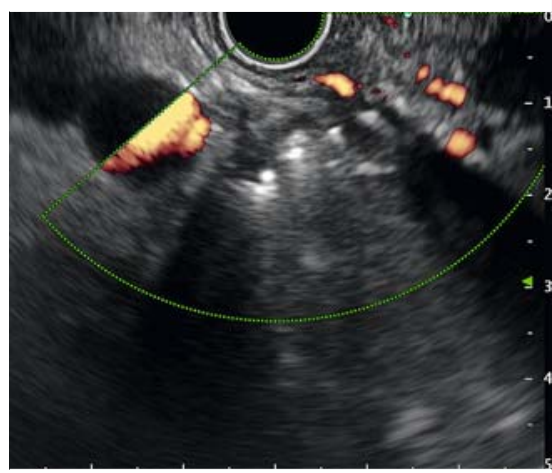

Fig. 3 Endoscopic ultrasound revealed complete occlusion of the aneurysm at the 16 -week follow-up.

\section{Competing interests}

The authors declare that they have no conflict of interest.

Hongtan Chen ${ }^{1}$, Feng $\mathrm{Li}^{2}$, Fenming Zhang ${ }^{1}$, Jianan Guo', Chunhui $\mathrm{Nie}^{3}$, Junhui Sun ${ }^{3}$, Guoqiang $\mathrm{Xu}^{1}$

1 Department of Gastroenterology, The First Affiliated Hospital, College of Medicine, Zhejiang University, Hangzhou, China

2 Department of Gastroenterology, Christus Trinity Mother Frances Hospital and Clinic, Tyler, Texas, USA

3 Department of Hepatopancreatobiliary China

\section{Corresponding author}

\section{Guoqiang Xu, MD, PhD}

Department of Gastroenterology, The First
[1] Hogendoorn W, Lavida A, Hunink MG et al. Open repair, endovascular repair, and conservative management of true splenic artery aneurysms. J Vasc Surg 2014; 60: 16671676

[2] Marone EM, Mascia D, Kahlberg A et al. Is open repair still the gold standard in visceral artery aneurysm management? Ann Vasc Surg 2011; 25: 936-946

[3] Gamanagatti S, Thingujam U, Garg P et al. Endoscopic ultrasound guided thrombin injection of angiographically occult pancreatitis associated visceral artery pseudoaneurysms: case series. World J Gastrointest Endosc 2015; 25: 1107-1113

[4] Tekola BD, Arner DM, Behm BW. Coil migration after transarterial coil embolization of a splenic artery pseudoaneurysm. Case Rep Gastroenterol 2013; 7: 487-491

[5] Frandah WM, Fiore N, Sherif M et al. Endoscopic diagnosis and management of chronic relapsing pancreatitis due to eroded embolization coils. Endoscopy 2020. doi:10.1055/a-1163-7195 Surgery, The First Affiliated Hospital, College of Medicine, Zhejiang University, Hangzhou, Affiliated Hospital, College of Medicine, Zhejiang University, 79 Qingchun Road, Hangzhou, China, 310003 Fax: +86-571-87236730 xugq@zju.edu.cn

\section{Bibliography}

Endoscopy 2021; 53: E194-E195

DOI 10.1055/a-1216-0670

ISSN 0013-726X

published online 2.9.2020

(C) 2020. Thieme. All rights reserved.

Georg Thieme Verlag KG, Rüdigerstraße 14, 70469 Stuttgart, Germany

\section{ENDOSCOPY E-VIDEOS}

https://eref.thieme.de/e-videos

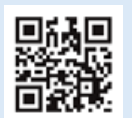

Endoscopy E-Videos is a free access online section, reporting on interesting cases and new techniques in gastroenterological endoscopy. All papers include a high quality video and all contributions are freely accessible online.

This section has its own submission website at https://mc.manuscriptcentral.com/e-videos 